\title{
DISTRIBUTION, DETECTION OF ENTEROTOXIGENIC STRAINS AND ANTIMICROBIAL DRUG SUSCEPTIBILITY PATTERNS OF BACTEROIDES FRAGILIS GROUP IN DIARRHEIC AND NON-DIARRHEIC FECES FROM BRAZILIAN INFANTS
}

\section{Débora Paula Ferreira ${ }^{1}$; Vânia Lúcia Silva ${ }^{1}$; Danielle Aparecida Guimarães ${ }^{1}$; Cíntia Marques Coelho²; Danielle Alves Gomes Zauli³ Luiz Macêdo Farias ${ }^{3}$; Maria Auxiliadora Roque Carvalho³ ${ }^{3}$ Claudio Galuppo Diniz ${ }^{1 *}$}

${ }^{1}$ Laboratório de Fisiologia e Genética Molecular Bacteriana, Departamento de Parasitologia, Microbiologia e Imunologia, Instituto de Ciências Biológicas, Universidade Federal de Juiz de Fora, Juiz de Fora, MG, Brasil; ${ }^{2}$ Laboratório de Ecologia Microbiana e Biologia Molecular, Departamento de Biologia, Instituto de Ciências Biológicas, Universidade Federal de Juiz de Fora, Juiz de Fora, MG, Brasil; ${ }^{3}$ Departamento de Microbiologia, Instituto de Ciências Biológicas, Universidade Federal de Minas Gerais, Belo Horizonte, MG, Brasil.

\begin{abstract}
Despite the importance of gastrointestinal diseases and their global distribution, affecting millions of individuals around the world, the role and antimicrobial susceptibility patterns of anaerobic bacteria such as those in the Bacteroides fragilis group (BFG) are still unclear in young children. This study investigated the occurrence and distribution of species in the BFG and enterotoxigenic strains in the fecal microbiota of children and their antimicrobial susceptibility patterns. Diarrheic $(n=110)$ and non-diarrheic $(n=65)$ fecal samples from children aged 0-5 years old were evaluated. BFG strains were isolated and identified by conventional biochemical, physiological and molecular approaches. Alternatively, bacteria and enterotoxigenic strains were detected directly from feces by molecular biology. Antimicrobial drug susceptibility patterns were determined by the agar dilution method according to the guidelines for isolated bacteria. BFG was detected in $64.3 \%$ of the fecal samples (55\% diarrheic and $80.4 \%$ non-diarrheic), and 4.6\% were enterotoxigenic. Antimicrobial resistance was observed against ampicillin, ampicillin/sulbactam, piperacillin/tazobactam, meropenem, ceftriaxone, clindamycin and chloramphenicol. The data show that these bacteria are prevalent in fecal microbiota at higher levels in healthy children. The molecular methodology was more effective in identifying the B. fragilis group when compared to the biochemical and physiological techniques. The observation of high resistance levels stimulates thoughts about the indiscriminate use of antimicrobial drugs in early infancy. Further quantitative studies are needed to gain a better understanding of the role of these bacteria in acute diarrhea in children.
\end{abstract}

Key words: Bacteroides fragilis group, ETBF, antimicrobial susceptibility, diarrhea

\footnotetext{
*Corresponding Author. Mailing address: Laboratory of Bacterial Physiology and Molecular Genetics, Department of Parasitology, Microbiology and Immunology, Federal University of Juiz de Fora, 36.036-900, Juiz de Fora, MG, Brazil.; Tel/Fax: + 55323229 32 13.;E-mail: claudio.diniz@ufjf.edu.br
} 


\section{INTRODUCTION}

The importance of anaerobic bacteria in the etiology of human gastrointestinal infections is well known $(30,39)$. Among these microorganisms, the ten related species of Gramnegative rods of the Bacteroides fragilis group (BFG) stand out. Though taxonomic alterations have been proposed, the group designation Bacteroides fragilis is still used (39). In spite of the protective function of these resident microorganisms, they can be associated with diseases in the gastrointestinal tract, mainly in situations of acute diarrhea and inflammatory intestinal diseases, among others (30).

In developing countries, these diseases are some of the most important causes of morbity and especially mortality, in children of low age (13). However, many cases of diarrhea are not diagnosed, due to mild and self-limited manifestations for which the patients do not seek medical service, or because there is an overload on the public health services, which makes the use of the available medical and laboratory resources difficult (21). Furthermore, there is a lack of scientific data on the incidence and the epidemiology of these diseases, which may interfere with public health policies, especially in developing countries.

Anaerobic bacteriology demands special routines with regards the collection, transport and processing of biological materials for studies involving the isolation and identification of these microorganisms (27). In this sense, the correct handling of the clinical specimens is essential for diagnosis and epidemiological research (15). The search for new methods of study may provide more exact knowledge of the participation of these microorganisms in different ecosystems (6).

Strains of $B$. fragilis eliciting intestinal secretions are named enterotoxigenic B. fragilis (ETBF) and their involvement as etiological agents of gastrointestinal diseases has been highlighted by several scientists in recent years $(28,34)$. Epidemiological data suggest that ETBF strains are globally distributed enteric pathogens that cause gastrointestinal disorders in humans, mainly young children. In addition, other animals like calves, lambs, foals, and piglets are susceptible to ETBF-associated diarrheal illnesses. Similar to the case for other enteric pathogens, asymptomatic ETBF colonization is common (34). This enterotoxin is associated with fluid secretion, exfoliation and alteration of the cytoskeleton of intestinal cells (22) and with the clinical demonstration of colorectal cancer (38).

In the last 20 years, geographical variations and an increase in resistance to antimicrobial drugs have been reported as a global problem. Periodic monitoring in these organisms of the patterns of susceptibility to drugs has been recommended to enable the appropriate choice of antibiotic therapy to be made $(17,24,36)$.

Regional studies about the incidence and characterization of Bacteroides spp., either enterotoxigenic or not, and their drug susceptibility patterns might provide knowledge of the distribution and the involvement of these bacteria in the diseases related to the gastrointestinal tract in different regions. Young individuals should be especially considered because their susceptibility to the complications related to the clinical demonstrations of gastrointestinal tract diseases is greater. The objective of this study was to evaluate the distribution of species of BFG in fecal samples from children with and without clinical demonstration of diarrheal disease by microbiological culture dependent methodology and/or molecular biology, their antimicrobial drug susceptibility patterns and the presence in the fecal samples of enterotoxigenic strains.

\section{MATERIAL AND METHODS}

\section{Specimen collection and microbiological culture}

One hundred and seventy five fecal samples were obtained (110 diarrheic and 65 non-diarrheic) from children (0 to 5 years of age) in Juiz de Fora, MG, Brazil. The study was approved by the Committee of Ethics on Research of the Federal University of Juiz de Fora. A brief epidemiological survey was also applied to assess the clinical status of each child, the sanitary living conditions, and to reject those who had undergone antimicrobial chemotherapy during the last 30 days. 
The feces, in natura, were collected in disposable sterilized bottles containing pre-reduced saline solution $(\mathrm{NaCl} 0.25 \%$, glycerine $10 \%$, L-cysteine $0.1 \%$, agar $0.5 \%$ ) and sent to the Laboratory of Bacterial Physiology and Molecular Genetics, with directions that the time between the collection and reception in the laboratory were critical for the research. The samples suitable for microbiological culture (up to 4 hours between collection and processing) were processed for isolation of BFG in Bacteroides Bile Esculin (BBE, HiMedia Laboratories) supplemented with gentamycin $100 \mu \mathrm{g} / \mathrm{mL}$, under anaerobiosis $\left(\mathrm{N}_{2} 90 \%\right.$ and $\left.\mathrm{CO}_{2} 10 \%\right)$. All the fecal samples including those not suitable for microbiological culture were diluted in saline solution and frozen at $-20^{\circ} \mathrm{C}$ for direct BFG detection.

\section{Conventional biochemical/physiological identification of bacterial samples}

Bacterial strains representative of BFG were isolated from BBE plates and were biochemically and physiologically identified according to established methods (37). The identification chart included verification of anaerobic physiology by respiratory test, bacterial morphology by Gram stain, determination of esculin hydrolysis, indole, catalase and sulfidric acid production, and carbohydrate fermentation (arabinose, celobiose, ramnose, sucrose, trealose, xilan, xylose, salicin). The tests, performed in duplicate, were repeated three times to validate the conventional biochemical/physiological identification.

\section{Molecular identification of the isolated strains and bacterial detection in feces}

Genomic DNA from the isolated bacterial strains and the total DNA present in the fecal specimens were extracted by chemical digestion with phenol-chloroform, according to previously described methods (6) and used as template in different multiplex polymerase chain reactions (PCR) specific for Bacteroides fragilis group identification (17). To ensure that bacterial identification and detection were not based on nonspecific DNA amplification the reactions were performed in triplicate. The reference strains $B$. caccae ATCC 43185, B. distasonis VPI 4223, B. eggerthii VPI B851, B. fragilis ATCC 25285, B. merdae ATCC 43184, B. ovatus VPI 0435, B. stercoris ATCC 43183, B. thetaiotaomicron ATCC 29148, B. uniformis VPI 0061, and B. vulgatus VPI 4245 were used as positive controls. A ubiquitous primer pair targeting bacterial DNA (341F 5'-CCTACGGGAGGCAGCAG-3' and 1391R 5'GACGGGCGGTGTGTRCA-3') was also used as an internal positive control with the following amplification conditions: initial denaturation $94{ }^{\circ} \mathrm{C}, 5 \mathrm{~min}$, followed by 30 cycles at 94 ${ }^{\circ} \mathrm{C}, 1 \mathrm{~min} ; 55^{\circ} \mathrm{C}, 1 \mathrm{~min} ; 72{ }^{\circ} \mathrm{C}, 2 \mathrm{~min}$, followed by a final extension of $72{ }^{\circ} \mathrm{C}, 10 \mathrm{~min}$ with a $1067 \mathrm{bp}$ amplicon expected. The negative control was performed in amplification reactions without the DNA template.

\section{Detection of enterotoxigenic Bacteroides fragilis (ETBF) in}

\section{the fecal specimens}

The ETBF was directly detected in the fecal samples by PCR according to a previously established procedure (28), with $0.5 \mu \mathrm{M}$ of the primers BF1 and BF2, and a 294 bp fragment was amplified. The enterotoxigenic B. fragilis ATCC 43859 was used as a positive control in these experiments.

\section{Antimicrobial drug susceptibility assays}

The minimum inhibitory concentration (MIC) was determined by the agar dilution method, according to the NCCLS guidelines (23) specific for anaerobe susceptibility testing. Antibiotic stock solutions were added to melted Brucella Agar (HiMedia Laboratories) to obtain final concentrations ranging from 0.06 to $1024 \mu \mathrm{g} / \mathrm{mL}$. The antimicrobial drugs were selected on the basis of microbial characteristics and clinical relevance: ampicillin, ampicillin/sulbactam, meropenem, piperacillin/tazobactam, ceftriaxone, clindamycin, chloramphenicol and metronidazole. The reference strain $B$. fragilis ATCC 25285 was included for quality control.

\section{RESULTS}

The fecal specimens were received and processed immediately, in the period from May 2007 up to December 
2008. The mean age of the donors was 26 months old. Approximately $52 \%$ of the samples were obtained in the rainy months from December to May, of which $50 \%$ were from patients with diarrhea and $55 \%$ from children from the control group. Regarding basic sanitation, $100 \%$ of the families had treated water available in their residences and drainage to a septic pit or sewer system.

Even though all collaborators had been instructed on the importance of sending the samples to the laboratory as quickly as possible for the bacteriology of anaerobes, there was great variation in the time from collection to processing, i.e. $23 \%$ of the samples were processed in the interval of up to four hours from collection and were considered suitable for cultivation, whereas $77 \%$ of the samples were processed more than four hours after collection (4 hours up to 9 days) for the direct detection of the $B$. fragilis group and ETBF strains by molecular biology. So, of the 175 fecal samples obtained, only 40 specimens were considered for isolation of 47 representative bacterial strains. The physiological/biochemical method allowed the identification of seven species of the group: $B$. distasonis (19.1\%), B. fragilis (17.0\%), B. vulgatus (17.0\%), B. caccae $(8.5 \%)$, B. thetaiotaomicron $(8.5 \%)$, B. uniformis $(2.1 \%)$ and $B$. merdae $(2.1 \%)$. The molecular biology method allowed the identification of four species of the group: $B$. vulgatus (44.7\%), B. fragilis (31.9\%), B. distasonis (14.9\%), and B. thetaiotaomicron (8.5\%). The two methodologies showed a discrepancy in the identification of 22 samples, which could be fitted into different taxonomic groups by the physiological/biochemical method or even classified into more than one taxonomic group (Table 1).

Table 1. Disagreements between molecular biology and conventional biochemical/physiological methods in identification of Bacteroides fragilis group species isolated from infantile diarrheic feces.

\begin{tabular}{|c|c|c|}
\hline \multirow[t]{2}{*}{ Strain code } & \multicolumn{2}{|r|}{ Bacterial identification methodology } \\
\hline & Molecular biology & Conventional biochemical/physiological \\
\hline FH4-1 & B. fragilis & B. distasonis \\
\hline FH5-5 & B. fragilis & B. distasonis \\
\hline FC4-6 & B. fragilis & B. merdae \\
\hline FH7-6 & B. fragilis & B. uniformis or B. vulgatus \\
\hline FH7-7 & B. fragilis & B. caccae or B. uniformis \\
\hline FH4-10 & B. fragilis & B. fragilis or B. uniformis or B. stercoris \\
\hline FH5-6 & B. fragilis & B. fragilis or B. distasonis or B. merdae \\
\hline FH9-11 & B. vulgatus & B. caccae \\
\hline FH10-1 & B. vulgatus & B. caccae \\
\hline FH10-7 & B. vulgatus & B. caccae \\
\hline FH10-12 & B. vulgatus & B. caccae \\
\hline FH10-16 & B. vulgatus & B. fragilis \\
\hline FH10-4 & B. vulgatus & B. uniformis \\
\hline FH9-7 & B. vulgatus & B. stercoris or B. vulgatus \\
\hline FH9-15 & B. vulgatus & B. vulgatus or B. stercoris \\
\hline FH10-9 & B. vulgatus & B. fragilis or B. stercoris \\
\hline FH10-13 & B. vulgatus & B. distasonis or B. caccae \\
\hline FH10-19 & B. vulgatus & B. stercoris or B. uniformis \\
\hline FH9-14 & B. vulgatus & B. merdae or B. caccae or B. stercoris \\
\hline FH10-3 & B. vulgatus & B. caccae or B. stercoris or B. uniformis \\
\hline FH10-10 & B. vulgatus & B. uniformis or B. stercoris or B. fragilis \\
\hline FH1-1 & B. distasonis & B. distasonis or B. caccae or B. merdae or B. thetaiotaomicron \\
\hline
\end{tabular}


When the evaluation was done directly by molecular biology, without microbiological culture, bacterial DNA was identified in $80 \%(n=140)$ of the fecal samples. Of the positive bacterial DNA samples, BFG species were detected in $64.3 \%$ $(n=90)$. Considering only the diarrheic feces, Bacteroides was detected in 55\% $(\mathrm{n}=49)$, whereas Bacteroides was detected in $80.4 \%(n=41)$ of the non-diarrheic feces, as shown in Table 2. In this study Bacteroides sp. were considered for the cases in which amplicons suggestive of Bacteroides were observed, but there was no amplification of fragments expected in the specific reactions even after repetition of the experiments.
ETBF was detected in $4.6 \%$ of the fecal specimens evaluated, four being in diarrheic fecal specimens (3.7\%) and four in nondiarrheic fecal specimens $(6.15 \%)$. No positive correlation was observed between ETBF detection and donor gender.

Regarding the antimicrobial drug susceptibility patterns of the BFG isolates, the tests revealed bacterial resistance to all with the exception of metronidazole. The least efficient drugs were: ampicillin, ceftriaxone, clindamycin, chloramphenicol and meropenem. On the other hand, the most efficient drugs were the compounds ampicillin/sulbactam and piperacillin/tazobactam as summarized in Table 3.

Table 2. Distribution of species within the Bacteroides fragilis group in diarrheic or non-diarrheic fecal samples from children aged 0-5 years old in Juiz de Fora, Brazil, detected directly by molecular biology, without microbiological culture.

\begin{tabular}{lcc}
\hline Bacterial identity & \multicolumn{2}{c}{ Frequency of detection (\%) } \\
\cline { 2 - 3 } & Diarrheic samples & Non-diarrheic samples \\
\hline Bacteroides fragilis & 22 & 32 \\
Bacteroides vulgatus & 31 & 36 \\
Bacteroides distasonis & 16 & 2 \\
Bacteroides thetaiotaomicron & 5 & 2 \\
Bacteroides cacae & 3 & - \\
Bacteroides ovatus & - & 3 \\
Bacteroides sp. & 23 & 25 \\
\hline
\end{tabular}

Table 3. Minimal inhibitory concentration (MIC) of antimicrobial drugs against the representative strains of the Bacteroides fragilis group isolated from infantile diarrheic feces in Juiz de Fora, Brazil.

\begin{tabular}{|c|c|c|c|c|c|c|}
\hline \multirow[t]{2}{*}{ Tested drugs } & \multicolumn{3}{|c|}{ MIC $(\mu \mathrm{g} / \mathrm{mL})$} & \multirow{2}{*}{$\begin{array}{l}\text { Susceptible } \\
\text { strains }(\%)\end{array}$} & \multirow{2}{*}{$\begin{array}{l}\text { Intermediary } \\
\text { resistance }(\%)\end{array}$} & \multirow{2}{*}{$\begin{array}{c}\text { Resistant } \\
\text { strains (\%) }\end{array}$} \\
\hline & $\mathbf{5 0 \%}$ & $90 \%$ & Range & & & \\
\hline Ampicillin & 2 & 32 & $0.06-512$ & 18.2 & 13.6 & 68.2 \\
\hline Ampicillin/sulbactam & 2 & 16 & $0.06-32$ & 84.1 & 4.5 & 11.4 \\
\hline Meropenem & 4 & 16 & $0.24-32$ & 54.5 & 27.3 & 18.2 \\
\hline Piperacillin/tazobactam & 4 & 16 & $0.48-128$ & 90.9 & 6.8 & 2.3 \\
\hline Ceftriaxone & 32 & 64 & $0.06-256$ & 40.9 & 38.6 & 20.5 \\
\hline Clindamycin & 4 & 8 & $0.24-16$ & 38.7 & 38.6 & 22.7 \\
\hline Chloramphenicol & 8 & 32 & $0.24-32$ & 50.1 & 34 & 15.9 \\
\hline Metronidazole & 0.06 & 2 & $0.06-2$ & 100 & - & - \\
\hline
\end{tabular}




\section{DISCUSSION}

The average low age of the population studied reflects the elevated frequency of acute diarrhea in children younger than two years of age and the gravity of the disease in this phase of life, which leads to the search for medical care $(6,25)$. The fecal specimens were obtained on dates distributed homogeneously in the rainy and dry months of the year. Most of the cases of diarrhea associated with bacteria occur in the rainy and hot months, possibly because the more elevated temperature favors bacterial multiplication in the environment, and the rain contributes to the dissemination of the etiological agent in the surface waters (31,32). Although the family income was not recorded, all the families had conditions of basic sanitation in their dwellings, such as treated water and a closed sewage system for feces.

Microbial cultivation allows the use of the isolates in subsequent investigations such as the determination of the antimicrobial drug susceptibility patterns and epidemiological studies. The Gram stain analysis and preliminary information on culture of the sample received can reveal the type and the relative number of microorganisms, as well as the host cells present, which can orient the clinical diagnosis and early therapy (29). The development of molecular methods allows the investigation, however, of microbial communities without the necessity for microbiological cultivation (15). When using the two methodologies, different situations were observed, with similar or divergent identifications. Some of the species of BFG are very similar biochemically, which makes the process of identification difficult (37), though these techniques are still widely used (5). In addition, the molecular technique allowed the detection of microorganisms in fecal samples, for which the method of classic microbiological culture cannot be applied or does not allow the recuperation of representative samples of BFG.

Both the isolated microorganisms and the genetic detection of the group directly in the fecal samples allowed the identification of representatives of the following BFG species: B. fragilis, B. distasonis, B. vulgatus, B. thetaiotaomicron, B. caccae and B. ovatus. The data are consistent with the literature, where the species most frequently isolated from infections or microbiota resident in healthy individuals of the human species are B. fragilis, B. thetaiotaomicron, $B$. distasonis and B. vulgatus (17).

Given that the BFG compose most of the anaerobic microbiota of the gastrointestinal tract, when the frequency of isolation or detection of these microorganisms is evaluated directly from the clinical specimens, at least two factors must be taken into account: (i) the low rate of isolation of Bacteroides in the feces can reflect the difficulties in recovering anaerobes in the laboratory, since the collection and adequate transport of specimens are crucial. (ii) Given the molecular methodology used and its basis on the detection of DNA sequences by polymerase chain amplification, it is possible that methodological failures could be associated with nonspecific amplification or even the lack of gene amplification due to the presence of substances inhibiting the DNA taq polymerase enzyme, as seen with the internal control with universal primers for bacterial DNA and as previously reported (1). On the other hand, given the frequency of these microorganisms, it is suggested that in fact a significant reduction of the BFG anaerobes in the fecal microbiota may take place in episodes of acute diarrhea. Little information is available on the resident microbiota during acute diarrhea in children (6), although a significant reduction of anaerobes, especially Bacteroides, is realized in acute diarrhea. These observations are attributed to the probable exacerbated growth of other microorganisms and alterations in the redox potential in the colon.

Enterotoxigenic strains were identified in $4.6 \%$ of the fecal samples. It is accepted that this number may vary from 6 to $12 \%$ of children in the USA, approximately $10 \%$ in Europe and around 2 to $15 \%$ in Asia $(9,11,25,29,34,35)$. In Brazil, there is a lack of scientific data on the incidence of ETBF in the population; however, data show the incidence of these microorganisms in HIV-positive children (3.1\%) (8) and immunocompetent children with diarrhea (1.5\% and $2.08 \%)$ $(5,16)$. The observation of ETBF in non-diarrheic fecal samples 
may indicate the presence of healthy bearers who serve as a reservoir of this microbial lineage for susceptible populations. It is known that environmental factors can modulate the profiles of gene expression in anaerobes, which might in these cases mean that toxins are not produced, but that the bacteria persist in the population $(6,10)$.

The observation of bacterial resistance to the tested antimicrobial drugs, with the exception of metronidazol, should be considered in empirical therapy, especially in clinical situations in which the characteristics of the disease could suggest the involvement of anaerobic bacteria, such as representatives of the BFG. Our data allow the suggestion of homogeneity in the drug susceptibility patterns between the different bacterial strains recovered. The high intermediary resistance to the antimicrobial drugs is significant and suggests a regional clinical alert. These microorganisms may represent the bacterial population circulating in the community, and children younger than 2 years old should not be expected to harbor resistant bacteria since they should not have been exposed to these drugs. Penicillins were observed to have low efficiency (ampicillin), followed by the cephalosporins (ceftriaxone) and carbapenems (meropenem). On the other hand, low levels of resistance were observed for betalactam/beta-lactamase inhibitor combinations, suggesting that most of the bacterial samples recovered may produce betalactamase enzymes, as already suggested for the BFG (33). The piperacillin-tazobactam association is still quite effective with low levels of resistance reported $(7,18)$. The resistance of the Bacteroides to penicillin was initially observed in 1966, and the production of different types of beta-lactamases represents the most important mechanism of resistance of these microorganisms to these drugs (14).

The increase in bacterial resistance to some antimicrobial agents, including tetracycline, clindamycin and ceftriaxone, reflects the capacity of $B$. fragilis to develop resistance through several different mechanisms $(2,7,13)$. Resistance among the Bacteroides isolates to clindamycin has been increasing in several countries in which epidemiological vigilance is more intense, mainly in the last two decades. This drug was considered the therapy of choice in anaerobic infections for a long period and today the rates of resistance may vary between $20-48 \%$ (18). Although in this study resistance to clindamycin has been verified at levels near to $35 \%$, in accordance with reports from other authors $(3,13,20)$, the studies of epidemiological vigilance do not describe with clarity the source of the bacterial samples. Given the source of the samples in our work, this number is significant. Besides, our results indicate high levels of intermediary resistance in the strains isolated from children without a recent history of therapeutic use of the antimicrobial drugs or different lincosamides.

The use of chloramphenicol has been declining in many countries due to the toxicity of the drug, which causes intestinal disorders, depression and anemia. On the other hand, in our country, this drug is consumed widely and in our study a high rate of resistance was observed for this antimicrobial $(7,12,26)$. According to the literature, in the regions where the drug is still used for the treatment of infections in which BFG is involved, levels of resistance to chloramphenicol are up to $11 \%$, though not all authors considered intermediary resistance $(7,20)$

In spite of the in vitro tests for the susceptibility of anaerobic bacteria that are not routinely considered for clinical decisions, some authors $(2,7,13,14,35,19)$ defend laboratory monitoring of the levels of microbial resistance for these bacterial groups, fundamentally for three reasons: (i) it is already well-documented that resistance to antimicrobial drugs by the Bacteroides has increased significantly, and their participation in endogenous polymicrobial infections has been recognized; (ii) a certain disparity exists between different regions and institutions regarding protocols and rules for the use of antimicrobial drugs; (iii) clinical studies show that the resistance to antimicrobial drugs of anaerobes like the Bacteroides spp. can be correlated with therapeutic clinical failure, resulting in increased morbidity and mortality. There are also limitations in the extrapolation between results of experiments in vitro and clinical reality in vivo (24).

The results presented in this work on the distribution, 
susceptibility patterns and methodological issues show the necessity of survey studies for clarifying the role of these microorganisms in the maintenance of health or the production of disease in human beings, along with studies of epidemiological vigilance for constant monitoring of their antimicrobial drug susceptibility patterns.

\section{ACKNOWLEDGEMENTS}

This study was supported by grants from the Fundação de Amparo à Pesquisa do Estado de Minas Gerais (FAPEMIG).

\section{REFERENCES}

1. Abu Al-Soud, W.; Râdström, P. (1998). Capacity of nine thermostable DNA polymerases to mediate DNA amplification in the presence of PCR-inhibiting samples. Appl. Environ. Microbiol. 64 (10), 3748-3753.

2. Aldridge, K.E.; Ashcraft, D.; Cambre, K.; Pierson, C.L.; Jenkins, S.G.; Rosenblatt, J.E. (2001). Multicenter survey of the changing in vitro antimicrobial susceptibility of clinical isolates of Bacteroides fragilis group, Prevotella, Fusobacterium, Porphyromonas, and Peptostreptococcus species. Antimicrob. Agents Chemother. 45 (4), 1238-1243.

3. Almeida, A.E.; Uzeda, M. (1987). Susceptibility to five antimicrobial agents of strains of the Bacteroides fragilis group isolated in Brazil. Antimicrob. Agents Chemother. 31 (4), 617-618.

4. Almeida, F.S.; Nakano, V.; Avila-Campos, M.J. (2007). Occurrence of enterotoxigenic and nonenterotoxigenic Bacteroides fragilis in calves and evaluation of their antimicrobial susceptibility. FEMS Microbiol. Lett. 272 (1), 15-21.

5. Antunes, E.N.F.; Ferreira, E.O.; Vallim, D.C.; Paula, G.R.; Seldin, L.; Sabra, A.; Ferreira, M.C.S.; Domingues, R.M.C.P. (2002). Pattern III non-toxigenic Bacteroides fragilis (NTBF) strains in Brazil. Anaerobe 8 (1), 17-22

6. Balamurugan, R.; Kumar, N.S.; Jayakanthan, K.; Pulimmod, A.; Puzazhendhi, S.; Ramakrishna, B.S. (2008). Probiotic administration alters the gut flora and attenuates colitis in mice administered dextran sodium sulfate. J. Gastroenterol. Hepatol. 23 (12), 1834-1839.

7. Betriu, C.E.; Gomez, M.; Lopes, F.; Rodrigues-Avial, I.; Piazo, J.J. (2008). Resistance trends of the Bacteroides fragilis group over a 10-year period, 1997-2006 in Madri, Spain. Antimicrob. Agents Chemother. 52 (7), 2686-2690.

8. Bressane, M.A.; Durigon, L.E.; Avila-Campos, M.J. (2001). Prevalence of the Bacteroides fragilis Group and Enterotoxigenic Bacteroides fragilis in Immunodeficient Children. Anaerobe 7 (5), 277-281.

9. Cohen, S.H.; Shetab, R.; Tang-Feldman, Y.J.; Sarma, P.; Silva Jr, J.; Prindiville, T.P. (2006). Prevalence of enterotoxigenic Bacteroides fragilis in hospital-acquired diarrhea. Diag. Microbiol. Infect. Dis. 55 (4), 251-254.

10. Diniz, C.G.; Farias, L.M.; Carvalho, M.A.R.; Rocha, E.R.; Smith, C.J. (2004). Differential gene expression in a B. fragilis metronidazoleresistant mutant. J. Antimicrob. Chemother. 54 (1), 100-108.

11. Durmaz, B.; Dalgalar, M.; Durmaz, R. (2005). Prevalence of enterotoxigenic B. fragilis in patients with diarrhea: a controlled study. Anaerobe 11 (6), 318-321.

12. Falagas, M.E.; Grammatikos, A.P.; Michalopoulos, A. (2008). Potential of old-generation antibiotics to address current need for new antibiotics. Expert Rev. Anti Infect. Ther. 6 (5), 593-600.

13. Fernández, C.L.; Castello, L.; Greco, G.; Legaria, M.C.; Litterio, M.; Predari, S.C.; Rollet, R.; Rossetti, A.; Carloni, G.; Sarchi, M.I.; Bianchini, H. (2007). Susceptibility trends of Bacteroides fragilis group isolates from Buenos Aires, Argentina. Rev. Argent. Microbiol. 39 (3), 156-160.

14. Fille, M.; Mango, M.; Lechner, M.; Schaumann, R. (2006). Bacteroides fragilis Group: Trends in Resistance. Curr. Microbiol. 52 (2), 153-157.

15. Furrie, M. (2006). A molecular revolution in the study of intestinal microflora. Gut 55 (2), 141-143.

16. Krzyzanowsky, F.; Avila-Campos, M.J. (2003). Detection of nonenterotoxigenic and enterotoxigenic Bacteroides fragilis in stool samples from children in São Paulo, Brazil. Rev. Inst. Med. Trop. Sao Paulo 45 (4), 225-227.

17. Liu, C.; Song, Y.; Mcteague, M.; Vu, A.W.; Wexler, H.; Finegold, S.M. (2003). Rapid identification of the species of the Bacteroides fragilis group by multiplex PCR assays using group- and species-specic primers. FEMS Microbiol. Lett. 222 (1), 9-16.

18. Liu, C.Y.; Huang, Y.T.; Liao, C.H.; Yen, L.C.; Lin, H.Y. (2008). Increasing trends in antimicrobial resistance among clinically important anaerobes and Bacteroides fragilis isolates causing nosocomial infections: emerging resistance to carbapenems. Antimicrob. Agents Chemother. 52 (9), 3161-3168.

19. Marina, M.; Ivanova, M.; Kantardjiev, T. (2009). Antimicrobial susceptibility of anaerobic bacteria in Bulgaria. Anaerobe 15 (4), 127132 .

20. Nakano, V.; Avila-Campos, M.J. (2004). Survey of antimicrobial susceptibility patterns of the bacteria of the Bacteroides fragilis group isolated from the intestinal tract of children. Mem. Inst. Oswaldo Cruz. 99 (3), 319-324.

21. Nakano, V.; Gomes, D.A.; Arantes, R.M.E.; Nicoli, J.R.; Avila-Campos, M.J. (2006). Evaluation of the pathogenicity of the Bacteroides fragilis toxin gene subtypes in gnotobiotic mice. Curr. Microbiol. 53 (2), 113117.

22. Nakano, V.; Gomes, T.A.T.; Vieira, M.A.M.; Ferreira, R.C.; AvilaCampos, M.J. (2007). bft gene subtyping in enterotoxigenic Bacteroides fragilis isolated from children with acute diarrhea. Anaerobe 13 (1), 1-5.

23. NCCLS (2004). M11-A6. In National Committee for Clinical Laboratory Standards. Reference method for Antimicrobial Susceptibility Testing for 
Anaerobic Bacteria.

24. Nguyen, M.H.; Yu, V.L.; Arthur, J.; Morris, A.J.; Dermott, L.M.; Wagner, M.W.; Snydman, D.R. (2000). Antimicrobial resistance and clinical outcome of Bacteroides bacteremia: findings of a multicenter prospective observational trial. Clin. Infect. Dis. 30 (6), 870-876.

25. Nguyen, T.V.; Van, P.L.; Huy, C.L.; Gia, K.N.; Weintraub, A. (2005). Etiology and epidemiology of diarrhea in children in Hanoi, Vietnam. Int. J. Infect. Dis. 10 (4), 298-308.

26. Páez, P.L.; Becerra, M.C.; Albesa, I. (2008). Chloramphenicol-induced oxidative stress in human neutrophils. Basic Clin. Pharmacol. Toxicol. 103 (4), 349-353.

27. Pang, X.; Ding, D.; Wei, G.; Zhang, M.; Wang, L.; Zhao, L. (2005) Molecular profiling of Bacteroides spp. in human feces by PCRtemperature gradient gel electrophoresis. J. Microbiol. Methods 61 (3), 413- 417.

28. Pantosti, A.; Malpeli, M.; Wilks, M.; Menozzi, M.G.; D’Ambrosi, F. (1997). Detection of enterotoxigenic Bacteroides fragilis by PCR. $J$. Clin. Microbiol. 35 (10), 2482-2486.

29. Pathela, P.; Hasan, K.Z.; Roy, E.; Alam, K.; Huq, F.; Siddique, A.K.; Sack, R.B. (2005). Enterotoxigenic Bacteroides fragilis - associated diarrhea in children 0-2 years of age in rural Bangladesh. J. Infect. Dis. 191 (8), 1245-1252.

30. Penders, J.; Thijs, C.; Vink, C.; Stelma, F.F.; Snijders, B.; Kummeling, I.; Van Den Brandt, P.A.; Stobberingh, E.E. (2006). Factors influencing the composition of the microbiota in early infancy. Pediatrics 118 (2), 511-521.

31. Podewils, L.J.; Mintz, E.D.; Nataro, J.P.; Parashar, U.D. (2004). Acute infectious diarrehea among children in developing countries. Semin. Pediatr. Infec. Dis. 15 (3), 155-168.
32. Quadri, F.; Svennerholm, A.M.; Faruque, A.S.G.; Sack, R.B. (2005). Enterotoxigenic Escherichia coli in developing countries: epidemiology, microbiology, clinical features, treatment and prevention. Clin. Microbiol. Rev. 18 (3), 465-483.

33. Salsbury, F.R.Jr; Crowder, M.W.; Kingsmore, S.F.; Huntley, J.J. (2009). Molecular dynamic simulations of the metallo-beta-lactamase from Bacteroides fragilis in the presence and absence of a tight-binding inhibitor. J. Mol. Model. 15 (2), 133-145.

34. Sears, C.L. (2009). Enterotoxigenic Bacteroides fragilis: a rogue among symbiotes. Clin. Microbiol. Rev. 22 (2), 349-369.

35. Sharma, N.; Chaudhry, R. (2006). Rapid detection of enterotoxigenic Bacteroides fragilis in diarrhoeal faecal samples. Indian J. Med. Res. 124 (5), 575-582.

36. Snydman, D.R.; Jacobus, N.V.; Mcdermott, L.A.; Ruthazer, R.; Golan, Y.; Goldestein, E.J.C.; Finegold, S.M.; Harrell, L.J.; Hecht, D.W.; Jenkins, S.G.; Pierson, C.; Venezia, R.; Yu, V.; Rihs, J.; Gorbach, S.L. (2007). National survey on the susceptibility of the Bacteroides fragilis group: report and analysis of the trends in the United States from 1997 to 2004. Antimicrob. Agents Chemother. 51 (5), 1649-1655.

37. Summanen, P.; Baron, E.J.; Citron, D.M.; Stron, C.A.; Wexler, H.M.; Finegold, S.M. (1993). Wadsworth Anaerobic Bacteriology Manual. Star Publishing Company, Belmont, California.

38. Toprak, N.U.; Yagci, A.; Gulluoglu, B.M.; Akin, M.L.; Demirkalem, P.; Celenk, T.; Soyletir, G. (2006). A possible role of Bacteroides fragilis enterotoxin in the aetiology of colorectal cancer. Clin. Microbiol. Infect. 12 (8), 782-786.

39. Wexler, H.M. (2007). Bacteroides: the good, the bad, and the nitty-gritty. Clin. Microbiol. Rev. 20 (4), 593-621. 